\title{
COMPACTNESS OF ISOSPECTRAL COMPACT MANIFOLDS WITH BOUNDED CURVATURES
}

\author{
Gengqiang Zhou
}

\begin{abstract}
Suppose that $\mathcal{I}^{n}(C)$ is the class of all Riemannian metrics on a given n-dimensional closed manifold such that their associated Laplacians (on functions) have the same spectrum by counting multiplicities and their sectional curvatures are uniformly bounded $|K| \leq C$ by a constant $C>0$. We show that the isospectral class $\mathcal{I}^{n}(C)$ is compact in the $C^{\infty}$-topology. This generalizes our previous $C^{\infty}$-compactness result, which holds for dimensions up to seven.
\end{abstract}

\section{Introduction.}

Let $M$ be a closed $C^{\infty}$-manifold which will be fixed throughout this paper as a fixed underlying manifold. A sequence of Riemannian metrics $\left\{g_{i}\right\}$ on $M$ converges in the $C^{k}$-topology if and only if there are diffeomorphisms $f_{i}: M \rightarrow M$, such that the metrics $\left\{f_{i}^{*} g_{i}\right\}$, as matrices in a smooth atlas of $\mathrm{M}$, converge in the $C^{k}$-topology on functions on domains in $\mathbf{R}^{n}$. Then, the $C^{\infty}$-topology will mean the topology on the space of metrics on a fixed manifold $M$, which may be defined via convergence of sequences in the $C^{k}$ topology for all $k$. Riemannian metrics $g_{1}$ and $g_{2}$ on $M$ are called isospectral if they have the same Laplacian spectrum by counting multiplicities. It was shown [15] that an isospectral set of closed Riemannian two manifolds is compact in the $C^{\infty}$-topology. In dimension three $C^{\infty}$-compactness results were proved for isospectral sets of metrics in a given conformal class of metrics [2], [5]. Most recently, R. Brooks, P. Perry and P. Petersen V. [3] proved that if $\left\{M_{i}\right\}$ is a family of isospectral manifolds of dimension $\mathrm{n}$ with negative sectional curvatures or sectional curvatures uniformly bounded from below, then $\left\{M_{i}\right\}$ contains only finitely many homeomorphism types. If $n \neq 4$, then $\left\{M_{i}\right\}$ contains only finitely many diffeomorphism types. In particular, when $n=3$, they proved that if $M_{i}$ all have negative sectional curvatures or have Ricci curvatures uniformly bounded from below, then $\left\{M_{i}\right\}$ is precompact in the $C^{\infty}$-topology. See also [1] for similar results. For $C^{\infty}$-compactness in higher dimensions, the author [17] has shown that $\mathcal{I}^{n}(C)$ is $C^{\infty}$-compact for $n \leq 7$. The author has learned that the same 
result was obtained by Professor R. Brooks and P. Chow. In this paper, we will generalize this result to arbitary dimension.

Theorem 1.1. Let $\mathcal{I}^{n}(C)$ be the class of all Riemannian metrics on a given n-dimensional closed manifold such that they have the same Laplacian spectrum by counting multiplicities and uniformly bounded sectional curvatures $|K| \leq C, C>0$. Then, the isospectral class $\mathcal{I}^{n}(C)$ is compact in the $C^{\infty}$-topology, for all $n$.

A direct consequnce of Theorem 1.1 is that the set, denoted by $\mathcal{I}^{n}$, of closed n-dimensional isospectral Riemannian manifolds is compact with respect to the Lipschitz topology. This is due to the fact that $\mathcal{I}^{n}$ contains only finite diffeomorphism types by Cheeger's finiteness theorem.

In the proof of Theorem 1.1, we fisrt obtain priori estimates on $\nabla^{k} R$ by using interpolation inequalities (see Proposition 3.1 below) and heat invariants. This in general gives better estimates than those given by combining the Sobolev embedding theorem and heat invariants. And then we obtain Theorem 1.1 by applying the $C^{k}$-version of the Cheeger-Gromov compactness theorem.

\section{Preliminaries.}

Let $H_{t}(x, y)$ be the fundamental solution to the heat equation

$$
\frac{\partial}{\partial t}+\Delta_{x}=0
$$

on $R^{+} \times M$. If we denote by $\left\{\lambda_{k}\right\}_{k \geq 0}$ the eigenvalues of the Laplacian $\Delta_{g}$. Then, the trace of $H_{t}(x, y)$ has the following asymptotic expansion:

$$
\begin{aligned}
Z(t) & =\operatorname{tr}\left(H_{t}\right)=\int_{M} H_{t}(x, x) d v_{g}(x)=\sum_{i=0}^{\infty} \exp ^{-\lambda_{i} t} \\
& \sim \frac{1}{(4 \pi t)^{\frac{n}{2}}}\left(a_{0}+a_{1} t+a_{2} t^{2}+\cdots\right),
\end{aligned}
$$

as $t \rightarrow 0^{+}$. The heat invariants $a_{k}$ as defined by the expansion are determined by the spectrum of $(M, g)$. Moreover, $a_{k}$ can be represented as integrals of expressions in the curvature tensor over $M$. In order to formulate these expressions, we introduce some notations. For a Riemannian metric $g=$ $\sum g_{i j} d x^{i} d x^{j}$, let $R$ denote the curvature tensor, $\rho=\rho_{i j}$ the Ricci tensor and $\tau$ the scalar curvature. Let $\left\{e_{i}\right\}$ be an orthonormal frame and ";" denote covariant differentiation. If $\left(n_{1}, n_{2}, \cdots, n_{s}\right)$ is a multiple index then 
we define:

$$
\begin{aligned}
\left|\nabla^{s} \tau\right|^{2} & =R_{i j i j ; n_{1} \cdots n_{s}} R_{k l k l ; n_{1} \cdots n_{s}}, \\
\left|\nabla^{s} \rho\right|^{2} & =R_{i k j k ; n_{1} \cdots n_{s}} R_{i l j l ; n_{1} \cdots n_{s}}, \\
\left|\nabla^{s} R\right|^{2} & =R_{i j k l ; n_{1} \cdots n_{s}} R_{i j k l ; n_{1} \cdots n_{s}},
\end{aligned}
$$

where we use the Einstein summation convention. Then, when $k=0,1,2,3$, the heat invariants are known as follows (cf. [9, p. 327]),

$$
\begin{aligned}
a_{0}= & \int_{M} 1=\operatorname{Vol}(M), \quad a_{1}=\frac{1}{6} \int_{M} \tau, \\
a_{2}= & \frac{1}{360} \int_{M} 5 \tau^{2}-2|\rho|^{2}+2|R|^{2}, \\
a_{3}= & \frac{1}{45360} \int_{M}\left(-142|\nabla \tau|^{2}-26|\nabla \rho|^{2}-7|\nabla R|^{2}+35 \tau^{3}-42 \tau \rho^{2}+42 \tau R^{2}\right. \\
& \left.-36 \rho_{i j} \rho_{j k} \rho_{k i}-20 \rho_{i j} \rho_{k l} R_{i k j l}-8 \rho_{i j} R_{i k l n} R_{j k l n}-24 R_{i j k l} R_{i j n p} R_{k l n p}\right) .
\end{aligned}
$$

In general, we have the following theorem which is due to Gilkey [8]. To state the theorem, we define that the weight of $R_{i j k l ; I}, I=\left(n_{1}, \cdots n_{s}\right)$ is $|I|+2=s+2$ and the weight of a monomial in such factors is the sum of the weights, where, in $R_{i j k l ; I}$.

Theorem 2.1. For $k \geq 3$,

$$
a_{k}=(-1)^{k} \int_{M} A_{k}\left|\nabla^{k-2} R\right|^{2}+B_{k}\left|\nabla^{k-2} \tau\right|^{2}+\int_{M} E_{k},
$$

where $A_{k}, B_{k}>0$ and $E_{k}$ is a polynomial of weight $2 k$ in contractions of $R_{i j k l ; I}$, with $|I| \leq k-3$.

Remark on Theorem 2.1. In his paper [8], Gilkey proves that

$$
a_{k}=(-1)^{k} \int_{M} A_{k}\left|\nabla^{k-2} \rho\right|^{2}+B_{k}\left|\nabla^{k-2} \tau\right|^{2}+\int_{M} E_{k} .
$$

Also he has observed that

$$
\int_{M}\left|\nabla^{s} R\right|^{2}=c_{1} \int_{M}\left|\nabla^{s} \rho\right|^{2}+c_{2} \int_{M}\left|\nabla^{s} \tau\right|^{2}+\cdots,
$$

where $c_{1}$ and $c_{2}$ are universal constants and "..." denotes lower order terms, so that (2.1) follows from (2.2) and (2.3). For the sake of completeness, we include a proof of (2.3) in Appendix, which is provided by Gilkey.

Now suppose $C>0, V>0, d>0$ are given constants. Let $\mathcal{M}(n, d, C, V)$ denote the class of all closed n-dimensional Riemannian manifolds $\mathrm{M}$ with 
diameter $\operatorname{diam}(M) \leq d$, volume $\operatorname{vol}(M) \geq V$ and sectional curvature $\left|K_{M}\right| \leq$ $C$. Then, $\left\{(M, g) \mid g \in \mathcal{I}^{n}(C)\right\}$ is a subclass of $\mathcal{M}(n, d, C, V)$ because the $\operatorname{vol}(M, g)$ is an isospectral invariant and the diameter of $(M, g)$ satisfies (cf. $[6])$

$$
\lambda_{i}(M) \leq \frac{(n-1)^{2}}{4} C+\frac{c_{n} i^{2}}{(\operatorname{diam}(M))^{2}}
$$

which implies that $\operatorname{diam}(M) \leq c\left(\lambda_{i}, n, C, i\right)$ when $i$ is large enough. Also, we recall the $C^{k}$-version of the Cheeger-Gromov compactness theorem ([10], [11], [16] and [14]).

Theorem 2.2. The space of n-dimensional Riemannian manifolds satisfying the following bounds:

$$
\left|\nabla^{j} R\right| \leq C, j \leq k, \quad \operatorname{vol}(M) \geq V, \quad \operatorname{diam}(M) \leq d,
$$

where covariant derivatives $\nabla^{j}$, volume and diameter are defined by individual Riemannain metric, consists of finitely many diffeomorphism types and any sequence in the space has a subsequence which converges with respect to the Lipschitz topology to an n-dimensional smooth limit manifold carrying a limit metric of Hölder class $C^{k+1, \alpha}$, for any $0<\alpha<1$.

\section{Compactness in $C^{\infty}$-topology.}

Let $T=\left\{T_{i_{1} \cdots i_{k}}\right\}$ be a tensor field on $(M, g)$. Denote

$$
|T|^{2}(x)=\langle T(x), T(x)\rangle=T_{i_{1} \cdots i_{k}} T_{j_{1} \cdots j_{k}} g^{i_{1} j_{1}} \cdots g^{i_{k} j_{k}},
$$

where $\langle\cdot, \cdot\rangle$ is the inner product on tensors with respect to the Riemannian metric. Then, we define

$$
\begin{aligned}
\|T\|_{\widehat{C}^{l}(M, g)} & =\sup _{x \in M, r \leq l}\left|\nabla^{r} T\right|(x), \\
\|T\|_{\widehat{W}^{l, p}(M)}^{p} & =\sum_{r \leq l} \int_{M}\left|\nabla^{r} T\right|^{p}(x) d v_{g}(x) .
\end{aligned}
$$

Since we will use Sobolev's embedding theorem in the following proofs, we wish to point out that Sobolev constants depend only on the zero-order information of metric ( for detailed discussions, cf. [4], [12]). Let $C_{I}(M)$ be the isoperimetric constant of $M$, i.e.

$$
C_{I}(M)=\inf _{S} \frac{\{\operatorname{area}(S)\}^{n}}{\left\{\min \left(\operatorname{vol}\left(M_{1}\right), \operatorname{vol}\left(M_{2}\right)\right)\right\}^{n-1}},
$$


where $S$ ranges over all compact $(n-1)$-dimensional submanifolds of $M$, dividing $M$ into two open submanifolds $M_{1}, M_{2}$ satisfying $\partial M_{1}=\partial M_{2}=S$. Also, let $C_{I}(\Omega)$ denote the isoperimetric constant of a domain $\Omega \subset M$ given by

$$
C_{I}(\Omega)=\inf _{S} \frac{\{\operatorname{area}(S)\}^{n}}{\{\operatorname{vol}(V)\}^{n-1}},
$$

where $S$ ranges over all compact $(n-1)$-dimensional submanifolds of $M$, contained in $\Omega$ and dividing $\Omega$ into a component $V$ which does not meet $\partial \Omega$, and other components. Note that if $\operatorname{vol}(\Omega) \leq \frac{1}{2} \operatorname{vol}(M)$, then $C_{I}(M) \leq$ $C_{I}(\Omega)$. Then, for all $f \in C_{0}^{\infty}(\Omega)$

$$
\left\{\int_{\Omega}|\operatorname{grad} f| d v\right\}^{n} \geq C_{I}(\Omega)\left\{\int_{\Omega}|f|^{\frac{n}{n-1}} d v\right\}^{n-1}
$$

and an argument similar to the proof of theorem 7.10 (Sobolev inequalities) of $[12]$ shows

$$
\|f\|_{L^{\frac{n p}{n-p}}(\Omega)} \leq c_{1}\|\operatorname{grad} f\|_{L^{p}(\Omega)}, \quad 1 \leq p<n
$$

and

$$
\sup _{\Omega}|f| \leq c_{2}\|\operatorname{grad} f\|_{L^{p}(\Omega)}, \quad p>n,
$$

where $c_{1}, c_{2}$ have upper bounds which depends only on $p$, lower bound of $C_{I}(\Omega)$ and $\operatorname{vol}(M)$. By choosing $\Omega$ so that $\operatorname{vol}(\Omega) \leq \frac{1}{2} \operatorname{vol}(M)$, we know that $c_{1}, c_{2}$ have upper bounds which depends only on $p$, a lower bound of $C_{I}(M)$ and $\operatorname{vol}(M)$. We notice that, by a theorem of C. Croke [7], $C_{I}(M)$ is bounded below by diameter, volume and Ricci curvature of $M$. So, $C_{I}(M, g), g \in$ $\mathcal{I}^{n}(C)$ are uniformly bounded from below. It follows by Theorem 2.2 that we may cover $M$ by geodesic balls with uniformly controled radii and number of balls and each geodesic ball has volume less than $\frac{1}{2} \operatorname{vol}(M)$. Thus, by an argument of partition of unity, we obtain,

$$
\|f\|_{L^{\frac{n p}{n-p}}(M)} \leq c_{1}^{\prime}\left(\|f\|_{L^{p}(M)}+\|\operatorname{grad} f\|_{L^{p}(M)}\right), \quad 1 \leq p<n
$$

and

$$
\sup _{M}|f| \leq c_{2}^{\prime}\left(\|f\|_{L^{p}(M)}+\|\operatorname{grad} f\|_{L^{p}(M)}\right), \quad p>n,
$$

and Sobolev constants $c_{1}^{\prime}, c_{2}^{\prime}$ are bounded uniformly with respect to metrics in $\mathcal{I}^{n}(C)$. By iterating the results above, we obtain inequalities for general cases. e.g.

$$
\sup |f| \leq c_{3}\|f\|_{W^{2, p}}, \quad p>\frac{n}{2} .
$$


For tensor $T$ on $(M, g)$, let $u(x)=|T(x)|=\langle T(x), T(x)\rangle^{1 / 2}$. Then, the weak gradient of $u(x)$ is given by

$$
\nabla u(x)= \begin{cases}|T(x)|^{-1}\langle\nabla T(x), T(x)\rangle, & |T(x)| \neq 0, \\ 0, & T(x)=0 .\end{cases}
$$

Thus, by Schwarz inequality,

$$
|\nabla u(x)| \leq|\nabla T(x)| .
$$

Hence, applying the above arguments to $u(x)$, we obtain that, for tensors, the Sobolev constants are also bounded uniformly with respect to metrics in $\mathcal{I}^{n}(C)$.

In order to show that the space $\mathcal{I}^{n}(C)$ is compact in the $C^{\infty}$-topology, we would like to show first that, for all $g \in \mathcal{I}^{n}(C), \widehat{W}^{k, 2}(M, g)$-norm of sectional curvature $R(g)$ is uniformly bounded for each $k \in \mathbf{Z}^{+}$. This implies that $\widehat{C}^{l}(M, g)$-norms of $R(g)$ for each $l \in \mathbf{Z}^{+}$and $g \in \mathcal{I}^{n}(C)$ are uniformly bounded since Sobolev constants are bounded. Then, we will show that $\mathcal{I}^{n}(C)$ is compact in the $C^{\infty}$-topology by using the $C^{k}$-version of the CheegerGromov compactness theorem.

Let us begin with introducing some notations. We know that in the expression of heat invariant $a_{k}, E_{k}$ is a polynomial of $R_{*, I},(|I| \leq k-3)$, under contractions, where "*" denotes component subindices of $R$. Then, a monomial $m$ in $E_{k}$ will be said of type $\left(k_{1}, \cdots, k_{i}\right)$ if it has the form $C R_{* ; l_{1}} \cdots R_{* ; l_{i}}$, where $\left|l_{1}\right|=k_{1}, \cdots,\left|l_{i}\right|=k_{i}$ and $C$ is a constant which may be different from term to term. Also, we will use the following propositions.

Proposition 3.1. ([13]). If $p \geq 1$, we have

$$
\begin{gathered}
\left\{\int_{M}|\nabla T|^{2 p} d v\right\}^{1 / p} \leq(2 p-2+n) \max _{M}|T|\left\{\int_{M}\left|\nabla^{2} T\right|^{p} d v\right\}^{1 / p}, \\
\int_{M}\left|\nabla^{i} T\right|^{2 k / i} d v \leq C(k, n) \max _{M}|T|^{2\left(\frac{k}{i}-1\right)} \int_{M}\left|\nabla^{k} T\right|^{2} d v
\end{gathered}
$$

for any tensor $T$ on $M$, where $n=\operatorname{dim} M$ and $1 \leq i \leq k, i \in \mathbf{Z}^{+}$.

Proposition 3.2. (Hölder inequality). If $u_{i} \in L^{p_{i}}, i=1, \cdots, l$, satisfy

$$
\frac{1}{p_{1}}+\cdots+\frac{1}{p_{l}}=1
$$

then

$$
\int u_{1} \cdots u_{l} d \mu \leq\left\|u_{1}\right\|_{p_{1}} \cdots\left\|u_{l}\right\|_{p_{l}}
$$


By Theorem 2.1, we have,

Lemma 3.1. For any metric $g \in \mathcal{I}^{n}(C)$, the following holds.

$$
\int_{M}\left|\nabla^{k} R\right|^{2} \leq c_{k+2}\left(\left|a_{k+2}\right|+\int_{M} E_{k+2}\right)
$$

where $k \geq 1, k \in \mathbf{Z}^{+}$and $E_{k+2}$ is a polynomial of weight $2(k+2)$ in contractions of $R_{i j k l ; I}$, with $|I| \leq k-1$.

Lemma 3.2. For each $k$, let

$$
N_{k}=\left\{\|R(g)\|_{\widehat{W}^{k, 2}(M, g)} \mid g \in \mathcal{I}^{n}(C)\right\} .
$$

Then, $N_{k}<+\infty$.

Proof. Lemma 3.2 will be proved by obtaining uniform $\widehat{W}^{k, 2}(M, g)$ estimates on $R(g)$ which will be proceeded by induction on $k$.

Because $R(g)$ is uniformly bounded and volume of $(M, g)$ is a spectral invariant, it is obviously that $\widehat{W}^{0,2}(M, g)$-norm of $R(g)$ is uniformly bounded.

When $k=1$, the explicit formula of the heat invariant $a_{3}$ in Section 1 implies

$$
\int_{M}|\nabla R(g)|^{2} d v(g) \leq C\left(a_{3}+\int_{M}|R(g)|^{3} d v(g)\right) .
$$

So the $\widehat{W}^{1,2}(M, g)$-norm of $R(g)$ is bounded by the assumption that curvatures are uniformly bounded. Thus, $N_{1}$ is bounded.

Now we assume that we have shown $\widehat{W}^{l, 2}(M, g)$-norms of $R(g), l<k$, are uniformly bounded. We would like to show that $R(g)$ is uniformly bounded in $\widehat{W}^{k, 2}(M, g)$-norm.

Again, $a_{k+2}$ is a spectral invariant. By Lemma 3.1, we need only obtain an upper estimate on $\int_{M} E_{k+2}$. It is known that $E_{k+2}$ has weight $2 k+4$. Let $m$ be a monomial of $E_{k+2}$. Then, $m$ must be of type:

$$
\left(k-\alpha_{1}, k-\alpha_{2}, \beta_{1}, \cdots, \beta_{j}\right)
$$

with $k-\alpha_{1} \geq k-\alpha_{2} \geq \beta_{1} \geq \cdots \geq \beta_{j} \geq 0$.

We first assume that $\beta_{j}>0$. By interpolation inequality (3.1), we known that if $R(g)$ is bounded in $\widehat{W}^{l, 2}(M, g)$, then $\widehat{W}^{i, 2 l / i}(M, g)$ norm of $R(g)$ is uniformly bounded, for $i \leq l, i \in \mathbf{Z}^{+}$. Now, we set $l=k-1$ and

$$
p_{1}=\frac{2(k-1)}{k-\alpha_{1}}, p_{2}=\frac{2(k-1)}{k-\alpha_{2}}, p_{3}=\frac{2(k-1)}{\beta_{1}}, \cdots, p_{j+2}=\frac{2(k-1)}{\beta_{j}} .
$$


In order to apply Hölder inequality (3.2) to $m$ we need only to prove

$$
\frac{1}{p_{1}}+\cdots+\frac{1}{p_{j+2}} \leq 1
$$

It is easy to get

$$
\frac{1}{p_{1}}+\cdots+\frac{1}{p_{j+2}}=\frac{2 k-\alpha_{1}-\alpha_{2}+\sum_{i=1}^{j} \beta_{i}}{2(k-1)}
$$

Then, (3.4) is equivalent to

$$
\sum_{i=1}^{j} \beta_{i} \leq \alpha_{1}+\alpha_{2}-2
$$

Recall that $E_{k+2}$ is of weight $2 k+4$. Then, by the very definition of weight, $m$ is of weight $2 k-\alpha_{1}-\alpha_{2}+4+2 j+\sum_{i=1}^{j} \beta_{i}$. Thus,

$$
\sum_{i=1}^{j} \beta_{i}=\alpha_{1}+\alpha_{2}-2 j
$$

This implies (3.5) since $j \geq 1$. Then (3.5) is true. Therefore, $m$ has bounded integral in this case by Hölder inequality.

If $\beta_{i}=0,1 \leq i \leq j$, then it is sufficient to consider monomial $m$ which is of type $(k-1, k-1,0)$. Obviously, $m$ has bounded integral by assumption.

Therefore $m$ has bounded integral. So is $\int_{M} E_{k+2} d v$. Thus, $\widehat{W}^{k, 2}(M, g)$ norm of $R(g)$ is uniformly bounded. Hence, by induction, $\widehat{W}^{k, 2}(M, g)$ norm of $R(g)$ is uniformly bounded for each $k$.

Proof of Theorem 1.1. By the previous discussions and Sobolev's embedding theorem, we conclude that $\widehat{C}^{l}(M, g)$-norms of $R(g)$ are uniformly bounded. Taking $i$ in (2.4) sufficiently large, we see that the diameter of $(M, g)$ is uniformly bounded by spectral data. Besides, volume is a spectral invariant. So conditions in (2.5) are satisfied. Thus, by Theorem $2.2, \mathcal{I}^{n}(C)$ is precompact in the $C^{\infty}$-topology. i.e., For any sequence $\left\{g_{k}\right\} \subseteq \mathcal{I}^{n}(C)$, there is a subsequence $g_{k_{j}}$ and diffeomorphisms $f_{k_{j}}$ on $M$ such that $f_{k_{j}}^{*}\left(g_{k_{j}}\right)$ converges to a smooth Riemannian metric $g_{\infty}$ on $M$. To show the smooth limit manifold $\left(M, g_{\infty}\right)$ has the same spectrum, we show that the heat kernel $H_{t}^{\infty}$ of $\left(M, g_{\infty}\right)$ has the same trace of heat kernel $H_{t}^{i}$, for each $i$, if $g_{i} \rightarrow g_{\infty}$ in the $C^{\infty}$-topology. Since $M$ is compact, then $H_{t}^{i} \rightarrow H_{t}^{\infty}, t>0$. Therefore, the trace of $H_{t}^{\infty}$ is the same as that of $H_{t}{ }^{i}$, for each $i$. So the values of $\lambda_{i}{ }^{\prime} s$ are determined. The multiplicity of each $\lambda_{i}$ is also determined because the $C^{\infty}$-convergence provide a uniform bound on the $C^{k}$-norms (with respect to 
a fixed metric $g_{0}$ ) on the solutions of $\Delta_{g} f_{g}^{i}=-\lambda_{i} f_{g}^{i}$, for each $\lambda_{i}$, (cf. [17]). Then $\left(M, g_{\infty}\right) \in \mathcal{I}^{n}(C)$.

Acknowledgement. The author wishes to thank Professor R. Strichartz for discussions in the writing of this paper. He would also like to thank Dr. Cao for his comments.

\section{Appendix.}

In a previous version of this paper, we proved Lemma 3.1 (in the case of $k \geq 2$ ) by proving the following Theorem A.1 before we saw a proof of (2.3). The proof of (A.1) is easy to follow and also (A.1) provides an estimate on $\int\left|\nabla^{k} R\right|^{2}$ by $\int\left|\nabla^{k} \rho\right|^{2}$ (only, without $\int\left|\nabla^{k} \tau\right|^{2}$ ) and lower order terms. So we include the proof of (A.1) in this appendix in addition to the proof of (2.3).

Theorem A.1. For any metric $g_{i j}$ on $M$, the curvature tensor $R_{i j k l}$ satisfies, for $k>1, k \in \mathbf{Z}^{+}$,

$$
\int_{M}\left|\nabla^{k} R\right|^{2} \leq 32 \int_{M}\left|\nabla^{k} \rho\right|^{2}+\int_{M} F
$$

where $F$ is a polynomial in contractions of $R_{i j k l ; I},|I|<k$, with weight $2 k+4$.

Proof. In the following proof, we will use $F_{1}, F_{2}, \cdots$, to denote polynomials in contractions of $R_{i j k l ; I},|I|<k$. By using Bianchi identities, we may derive the following formula (see [13], for details). In a local orthonormal frame,

$$
\begin{aligned}
\Delta R_{i j k l}= & \nabla_{i} \nabla_{k} \rho_{j l}-\nabla_{i} \nabla_{l} \rho_{j k}-\nabla_{j} \nabla_{k} \rho_{i l}+\nabla_{j} \nabla_{l} \rho_{i k}+R_{p j k l} \rho_{p i}+R_{i p k l} \rho_{p j} \\
& +2\left(R_{p i q j} R_{p k q l}-R_{p i q j} R_{p l q k}-R_{p i q l} R_{p j q k}+R_{p i q k} R_{p j q l}\right)
\end{aligned}
$$

Let $I$ be a multi-index with $|I|=k-2$. Then, by exchanging order of differentiation,

$$
\Delta R_{i j k l ; I}=\nabla_{i} \nabla_{k} \rho_{j l ; I}-\nabla_{i} \nabla_{l} \rho_{j k ; I}-\nabla_{j} \nabla_{k} \rho_{i l ; I}+\nabla_{j} \nabla_{l} \rho_{i k ; I}+G,
$$

where $G$ is of order at most $k-2$. Then, by Schwarz inequality,

$$
\begin{aligned}
& \int_{M} \Delta R_{i j k l ; I} \Delta R_{i j k l ; I} \\
& \leq 2 \int_{M}\left|\nabla_{i} \nabla_{k} \rho_{j l ; I}-\nabla_{i} \nabla_{l} \rho_{j k ; I}-\nabla_{j} \nabla_{k} \rho_{i l ; I}+\nabla_{j} \nabla_{l} \rho_{i k ; I}\right|^{2}+G^{2} \\
& \leq 32 \int_{M}\left|\nabla^{k} \rho\right|^{2}+2 \int_{M} G^{2} .
\end{aligned}
$$


Also, we have

$$
\begin{aligned}
\int_{M} \Delta R_{i j k l ; I} \Delta R_{i j k l ; I} & =\int_{M} R_{i j k l ; I_{\alpha \alpha}} R_{i j k l ; I_{\beta \beta}} \\
& =-\int_{M} R_{i j k l ; I_{\alpha}} R_{i j k l ; I_{\beta \beta \alpha}} \\
& =-\int_{M} R_{i j k l ; I_{\alpha}} R_{i j k l ; I_{\beta \alpha \beta}}+F_{1} \\
& =-\int_{M} R_{i j k l ; I_{\alpha}} R_{i j k l ; I_{\alpha \beta \beta}}+F_{2} \\
& =-\int_{M} R_{i j k l ; I_{\alpha \beta}} R_{i j k l ; I_{\alpha \beta}}+F_{2} \\
& =\int_{M}\left|\nabla^{k} R\right|^{2}+F_{2} .
\end{aligned}
$$

Therefore,

$$
\int_{M}\left|\nabla^{k} R\right|^{2} \leq 32 \int_{M}\left|\nabla^{k} \rho\right|^{2}+\int_{M} F
$$

So Theorem A.1 is proved.

Gilkey's formula (2.3) obviously displays a more accurate relations between $R, \rho$ and $\tau$. The formula should be of other interests. What follows is a proof of (2.3) provided by Gilkey (up to minor changes). We thank Professor Gilkey for allowing the author to include his proof in this paper.

Lemma A.1. Let $\omega:=T_{i} e_{i}$ be a 1-form valued invariant. Then

$$
\int_{M} T_{i ; i}|d \operatorname{vol}|=0
$$

where we sum over indices in pairs.

Proof. This is Stokes Theorem. It uses the fact that the volume form is parallel; $T_{i ; i}$ is minus the divergence of $\omega$. More precisely, if $f$ is a scalar function and $\omega$ a 1 -form, then

$$
d f=f_{; i} e_{i} \quad \text { and } \quad \delta \omega=-\omega_{i ; i} .
$$

Lemma A.2. $\int R_{i j l n ; k} R_{i j k l ; n}=-\int R_{i j l n ; k n} R_{i j k l}=\int R_{i j l n ; n} R_{i j k l ; k}+\cdots$

Proof. Let $\omega=R_{i j l n ; k} R_{i j k l} e_{n}$. Thus $\omega_{n}=R_{i j l n ; k} R_{i j k l}$. By Lemma A.1,

$$
0=\int\left(R_{i j l n ; k} R_{i j k l}\right)_{; n}=\int\left(R_{i j l n ; k n} R_{i j k l}+R_{i j l n ; k} R_{i j k l ; n}\right) .
$$


Consequently

$$
\int R_{i j l n ; k} R_{i j k l ; n}=-\int R_{i j l n ; k n} R_{i j k l} .
$$

We switch indices and introduce a curvature term.

$$
\int R_{i j l n ; k n} R_{i j k l}=\int R_{i j l n ; n k} R_{i j k l}+\cdots
$$

We apply the same argument using Lemma A.1 to see

$$
\int R_{i j l n ; n k} R_{i j k l}=-\int R_{i j l n ; n} R_{i j k l ; k} .
$$

The desired result now follows.

Theorem A.2. Let $P$ be an integral invariant of order $2 n+4$ in the jets of the metric for $n \geq 1$. Then there exist universal constants a and $b$ so

$$
\int_{M} P(g) d x=a \int_{M}\left|\nabla^{n} \tau\right|^{2}+b \int_{M}\left|\nabla^{n} \rho\right|^{2}+\mathcal{E}
$$

where $\mathcal{E}$ involves cubic terms with fewer than $n$ covariant derivatives of the curvature tensor.

Proof. Here is a quick sketch. I am going to take $n=4$ for simplicity of notation; this is totally irrelevant to the proof. Working modulo lower order terms, we use H. Weyl's theorem ([10, p. 132]) to write $P$ as a sum of terms of the form

$$
R_{\ldots ; \ldots}, \ldots \ldots ; \ldots,
$$

where exactly three covariant derivatives appear in each term and where we sum over indices in pairs. Since we are integrating over a manifold without boundary, we can integrate by parts to exchange covariant derivatives; since we are working modulo lower order terms, we can commute covariant derivatives. We use these observations without further comment in what follows.

We wish to simplify things. Suppose first we are dealing with

$$
R_{i j k l ; \ldots .} R_{a b c d ; \cdots}
$$

where the indices $\{i, j, k, l\}$ are distinct and that $\{a, b, c, d\}$ is a permutation of $\{i, j, k, l\}$; i.e. all the indices appearing in the covariant derivatives are contracted in pairs and the indices $\{i, j, k, l, a, b, c, d\}$ are contracted in pairs. Then we are dealing with

$$
R_{i j k l ; \ldots .} R_{i j k l ; \ldots} \text { or } R_{i j k l ; \ldots .} R_{i k j l ; \ldots .}
$$


we use the first Bianchi identity to express the second in terms of the first and deal with

$$
R_{i j k l ; x \ldots} R_{i j k l ; x \ldots}
$$

We apply the second Bianchi identity and integrate by parts to express this in terms of

$$
R_{i j x k ; l \ldots} R_{i j k l ; x \ldots} \text { and } R_{i j l x ; k \ldots} R_{i j k l ; x \ldots}
$$

Suppose next the indices $\{i, j, k, l\}$ are distinct. By the previous paragraph, we may assume $i$ appears as a covariant derivative. By integrating by parts, we may assume we are dealing with

$$
R_{i j k l ; i \ldots R \ldots ; \ldots}
$$

We apply the second Bianchi identity to deal with

$$
R_{i j l i ; k \ldots} R_{\ldots ; \ldots} \text { and } R_{i j l i ; k \ldots} R_{\ldots ; \ldots}
$$

Thus we may assume that we are actually dealing with

$$
R_{i j i k ; \ldots} R_{a b c d ; \cdots}
$$

where possibly $j=k$. If the indices $\{a, b, c, d\}$ are all distinct, at most two can belong to $\{j, k\}$ and consequently at least one of them appears as a covariant derivative. Thus the same argument gives us a term like

$$
R_{i j i k ; \cdots . .} R_{a b c d ; a \cdots}
$$

Again, use of the second Bianchi identity permits us to reduce the study to

$$
R_{i j i k ; \cdots . .} R_{a b a d ; \cdots} .
$$

Suppose $j \neq k$. We note that $R_{i j i k ; k}=1 / 2 R_{i k i k ; j}$ so

$$
R_{i j i k ; k \cdots}=\frac{1}{2} R_{i k i k ; j \cdots}
$$

If $k$ appears as a covariant derivative, we are dealing with

$$
R_{i k i k ; \cdots . . .} R_{a b a d ; \cdots}
$$

If $b \neq d$, both $b$ and $d$ appear as covariant derivatives and we are dealing with

$$
R_{i k i k ; \cdots} R_{a b a b ; \cdots}
$$


and after integration by parts this is seen to be $\left|\nabla^{n} \tau\right|^{2}$. The only remaining case is that we are dealing with $R_{i j i k ; \ldots} R_{a j a k ; \ldots}$ and after integration by parts this is seen to be $\left|\nabla^{n} \rho\right|^{2}$ modulo lower order terms. Theorem A.2 is proved.

Here is the method for computing the universal constants.

$$
\begin{aligned}
\int\left|\nabla^{\nu} R\right|^{2} & =\int R_{i j k l ; n \ldots} R_{i j k l ; n \ldots} \\
& =\int\left\{-R_{i j l n ; k \ldots} R_{i j k l ; n}-R_{i j n k ; l \ldots} R_{i j k l ; n \ldots}\right\} \\
& =\int-2 R_{i j l n ; k \ldots} R_{i j k l ; n \ldots} \\
& =\int-2 R_{i j l n ; n \ldots} R_{i j k l ; k \ldots}+\ldots \\
& =\int\left\{2 R_{j n l n ; i \ldots} R_{i j k l ; k \ldots}+2 R_{n i l n ; j \ldots} R_{i j k l ; k \ldots}+\cdots\right\} \\
& =\int 4 R_{j n l n ; i \ldots} R_{i j k l ; k \ldots}+\ldots \\
& =\int-4 \rho_{j l ; i \ldots} R_{i j k l ; k \ldots+\ldots} \\
& =\int\left\{4 \rho_{j l ; i \ldots} R_{j k k l ; i \ldots}+4 \rho_{j l i ; \ldots} R_{k i k l ; j}+\ldots\right\} \\
& =\int\left\{4\left|\nabla^{\nu} \rho\right|^{2}-4 \rho_{j l ; i \ldots} \rho_{i l ; j \ldots}+\ldots\right\} \\
& =\int\left\{4\left|\nabla^{\nu} \rho\right|^{2}-4 \rho_{j l ; j \ldots} \rho_{i l ; i \ldots}+\cdots\right\} \\
& =\int\left\{4\left|\nabla^{\nu} \rho\right|^{2}-\left|\nabla^{\nu} \tau\right|^{2}+\ldots\right\} .
\end{aligned}
$$

\section{References}

[1] M.T. Anderson, Remarks on the compactness of isospectral set in low dimensions, Duke Math. J., 63 (1991), 699-711.

[2] R. Brooks, P. Perry and P. Yang, Isospectral sets of conformally equivalent metrics, Duke Math. J., 58 (1989), 131-150.

[3] R. Brooks, P. Perry and P. Petersen, Compactness and finiteness heorems for isospectral manifolds, J. reine angew. Math., 426 (1992), 67-89.

[4] I. Chavel, Eigenvalues in Riemannian geometry, Academic Press, 1984.

[5] S.Y.A. Chang and P. Yang, Isospectral conformal metrics on 3-manifolds, JAMS, 3 (1990), 117-145.

[6] S.Y. Cheng, Eigenvalue comparison theorems and its geometric applications, Math. Zeit., 143 (1975), 289-297. 
[7] C.B. Croke, Some isoperimetric inequalities and eigenvalue estimates, Ann. Scient. Éc. Norm. Sup., 13 (1980), 419-435.

[8] P.B. Gilkey, Heat equation asymptotics, Proc. of Symp. in Pure Math., 54 (1993), Part 3, 317-326.

[9] Leading terms in the asymptotics of the heat equation, Contemporary Math., 73 (1988), 79-85.

[10] Invariance theory, the heat equation, and the Atiyah-Singer index theorem, Publish or Perish, Inc., 1984.

[11] M. Gromov, Structures métriques pour les variétés riemanniennes, rédigé par J. Lafontaine et P. Pansu, Cedic-Nathan, 1980.

[12] R.E. Greene and H. Wu, Lipschitz convergerce of Riemannian manifolds, Pacific J. Math., 131(1) (1988), 119-141.

[13] D. Gilbarg and N.S. Trudinger, Elliptic partial differential equations of second order, Springer-Verlag, 1983.

[14] R.S. Hamilton, Three-manifolds with positive Ricci curvature, J. Diff. Geom., 17 (1982), 255-306.

[15] A. Kasue, A convergence theorem for Riemannian manifolds and some applications, Nagoya Math. J., 114 (1989), 21-51.

[16] B. Osgood, R. Phillips and P. Sarnak, Compact isospectral sets of Riemann surfaces, J. Funct. Anal., 80 (1988), 212-234.

[17] S. Peters, Convergence of Riemannian manifolds, Comp. Math., 62 (1987), 3-16.

[18] G. Zhou, Compactness of isospectral compact manifolds of bounded curvatures up to dimensions seven, preprint.

Received October 1, 1993 and revised November 18, 1994.

MSRI

BERKELEy, CA 94720

E-mail address: gzhou@msri.org 